\title{
Propriedades psicométricas da Escala de Graus de Doença Determinados pelo Paciente com Esclerose múltipla
}

\author{
Psychometric properties of the Degree of Disease Scale \\ Determined by the Patient with Multiple Sclerosis
}

\author{
Propiedades psicométricas de la Escala de grados de \\ enfermedad determinada por el paciente con esclerosis \\ múltiple
}

\section{Leilane Maria Quaresma da Silva ${ }^{1}$, Milene Soares Nogueira de Lima ${ }^{2}$ Clarissa Cardoso dos Santos-Couto-Paz ${ }^{3}$}

1.Fisioterapeuta, Mestra do Programa de Pós-Graduação em Ciências e Tecnologias da Saúde, Universidade de Brasília (UnB), Brasília-DF, Brasil.

2. Fisioterapeuta, Mestra em Educação Física pela Universidade de Brasília (UnB), Doutoranda do Programa de Pós-Graduação em Ciências e Tecnologias da Saúde, Universidade de Brasília (UnB), Brasília-DF, Brasil.

3. Fisioterapeuta, Professora do Programa de Pós-Graduação em Ciências e Tecnologias da Saúde da Faculdade de Ceilândia, Professora Adjunta da Universidade de Brasília, Brasília-DF, Brasil.

\begin{abstract}
Resumo
Objetivo. Identificar se a Escala de Graus de Doença Determinados pelo Paciente (PDDS) possui os parâmetros psicométricos adequadas para sua aplicação clínica em comparação com a Escala Expandida do Estado de Incapacidade (EDSS) em indivíduos com esclerose múltipla. Método. Foi realizada uma busca integrativa da literatura nas bases de dados MEDLINE (PubMed), PEDRo e Livivo produzindo 373 resultados. Publicações de texto completo relevantes foram identificadas usando resumo e, em seguida, revisões de texto completo e a literatura foi revisada. Resultados. Para avaliação das propriedades psicométricas (validade, confiabilidade, reprodutibilidade) da PDDS, foram identificadas 7 publicações de texto completo relevantes. A PDDS possui forte correlação com a EDSS, além de boa confiabilidade e reprodutibilidade. Além disso, possui tradução para o espanhol, português e italiano, considerada, portanto, uma escala confiável, que quantifica a incapacidade dos indivíduos e avalia a progressão da doença, porém com um enfoque principalmente na deambulação. Conclusão. A PDDS atende aos padrões metodológicos de validade da escala e é uma alternativa viável para a complementação da EDSS quando esta não puder ser aplicável na prática clínica.
\end{abstract}

Unitermos. Esclerose múltipla; Escala de Graus de Doença Determinados pelo Paciente; Escala expandida do estado de incapacidade; Propriedades psicométricas; Validade

\footnotetext{
Abstract

Objective. Identify whether the Patient Determined Disease Steps (PDDS) scale has the psychometric parameters required to validate it in relation to the Expanded Disability Status Scale (EDSS) in individuals with multiple sclerosis. Method. We have conducted an integrative literature search in the MEDLINE (PubMed), PEDRo and Livivo databases, yielding 373 results. Relevant full-text publications were identified using summary and full-text reviews, and the literature was reviewed. Results. With the aim of assessing the psychometric properties (validity, reliability, reproducibility) of the PDDS scale, seven relevant full-text publications were identified. The PDDS has a strong correlation with the EDSS, in addition to good reliability and reproducibility. Besides that, this scale makes translations for Spanish, Portuguese and Italian available. Thus, it is a reliable scale, which quantifies individual's incapacity and assesses the progression of the disease. However, it focuses mainly on ambulation.
} 
Conclusion. The PDDS scale meets the psychometric standards of scale validity and it is a viable alternative to complementing EDSS, when the latter cannot be applied.

Keywords. Multiple Sclerosis; Patient Determined Disease Steps; Expanded Disability Status Scale; Psychometric properties; Validity

\begin{abstract}
Resumen
Objetivo. Identificar si la Escala de grados de enfermedad determinados por el paciente con esclerosis múltiple (PDDS) tiene los parámetros psicométricos necesarios para su validación en comparación con la Escala Expandida del Estado de la Discapacidad (EDSS) en individuos con esclerosis múltiple. Método. Se realizó una búsqueda bibliográfica integradora en las bases de datos MEDLINE (PubMed), PEDRo y Livivo con 373 resultados. Las publicaciones relevantes de texto completo se identificaron mediante resúmenes y luego revisiones de texto completo, y se revisó la literatura. Resultados. Para evaluar las propiedades psicométricas (validez, confiabilidad, reproducibilidad) de la PDDS, se identificaron siete publicaciones de texto completo relevantes. La escala PDDS tiene una fuerte correlación com la escala EDSS, así como una buena fiabilidad y reproducibilidad. Además, se traduce al español, portugués e italiano, por lo que se considera una escala fiable que cuantifica la discapacidad de las personas y evalúa la progresión de la enfermedad, pero se centra principalmente en la deambulación. Conclusión. La escala PDDS cumple con los estándares psicométricos de validez de escala y es una alternativa viable para complementar la EDSS, cuando esta última no se pueda aplicar. Palabras clave. esclerosis múltiple; Escala de grados de enfermedad determinados por el paciente con esclerosis múltiple; Escala Expandida del Estado de la Discapacidad; Propiedades psicométricas; Validez
\end{abstract}

Trabalho realizado na Universidade de Brasília, Brasília-DF, Brasil.

\title{
INTRODUÇÃO
}

A Esclerose Múltipla (EM) é uma doença inflamatória crônica do Sistema Nervoso Central que provoca déficits neurológicos de forma irregular, cuja remissão é variável. A doença atinge com frequência indivíduos do sexo feminino de etnia branca, com idade entre 20 e 40 anos $^{1,2}$. A maioria dos indivíduos com EM (aproximadamente 80\%) inicialmente apresentam episódios de surtos-remissão ou remitente recorrente (EMRR), os quais são períodos em que ocorrem surtos de sintomas seguidos por períodos de remissão total ou parcial dos mesmos. Entretanto, após cerca de 10 a 15 anos, 60\% destes indivíduos podem 
manifestar uma transição da doença para o tipo progressiva secundária (EMPS), que é caracterizada por um declínio gradual da função neurológica ${ }^{3}$. Contudo, em menos de $15 \%$ dos casos, a doença pode se apresentar de imediato na forma de progressiva primária (EMPP) ${ }^{3}$.

A fim de entender e descrever a gravidade clínica e os déficits funcionais na EM, foram desenvolvidos vários instrumentos que são frequentemente utilizados como variável de desfecho primária em ensaios clínicos para avaliar a eficácia das intervenções terapêuticas ${ }^{4}$.

O instrumento mais amplamente utilizado é a Escala Expandida do Estado de Incapacidade (EDSS) de Kurtzke ${ }^{5}$, que é usada para descrever a progressão da doença em pacientes com EM e avaliar a eficácia de intervenções terapêuticas em ensaios clínicos ${ }^{5}$. Ela avalia nove sistemas funcionais do sistema nervoso central e a sua pontuação final consiste em uma classificação ordinal variando de 0 (estado neurológico normal) a 10 (morte por EM), com intervalos de 0,5 de incrementos (ao atingir o EDSS 1). Entretanto, seu escore final é baseado principalmente na necessidade de auxílio para a deambulação sendo que, em indivíduos com comprometimento leve, o escore da EDSS varia entre 0 e 4; comprometimento moderado, EDSS entre 4,5 e 6; e comprometimento grave, EDSS 6,5 a 9,5.

As escalas clínicas atuais para avaliação de pessoas com diagnóstico de EM apresentam algumas características que dificultam sua aplicação clínica, incluindo necessidade de treinamento intensivo para administração, influência da 
prática do avaliador, o que gera baixa confiabilidade intraexaminadores, falta de representação uniforme entre os graus, além disso, tais escalas são pouco sensíveis à progressão em determinados estágios da doença ${ }^{6}$. Por essas razões, a fim de avaliar melhor a incapacidade dos indivíduos com EM foi desenvolvida a Escala de Graus de Doença Determinados pelo Paciente (PDDS) pelos pesquisadores associados com o Registro de Pacientes do Comitê NorteAmericano de Pesquisa em EM (NARCOMS) ${ }^{6}$. Essa escala considera as incapacidades relatadas pelos pacientes de forma a substituir a EDSS. A PDDS tem nove níveis ordinais variando entre 0 (normal) e 8 (acamados) ${ }^{7}$ e os escores do PDDS podem ser convertidos em escores do EDSS $^{8}$ bem como classificações de incapacidades leves, moderadas ou graves $^{9}$. Contudo, não existe uma correspondência direta entre as pontuações.

Poucos instrumentos desenvolvidos para as incapacidades na EM possuem requisitos dos padrões metodológicos para uso em ensaios clínicos. Portanto, o objetivo deste estudo foi identificar se a escala PDDS possui propriedades psicométricas adequadas para sua aplicação clínica, incluindo validade e confiabilidade, além disso, analisar a correlação entre a PDDS e as principais escalas utilizadas como variável de desfecho em ensaios clínicos. 


\section{MÉTODO}

O método de revisão integrativa é uma abordagem que permite a inclusão simultânea de pesquisas experimentais e não experimentais, combinação de dados da literatura teórica e empírica, além de incorporar uma ampla gama de propósitos, como definir conceitos, revisar teorias ou evidências e analisar questões metodológicas de um tópico específico ${ }^{10}$. Por mais abrangente que seja a revisão integrativa, esta inclui uma abordagem sistemática e rigorosa do processo de revisão, principalmente na análise de dados com o intuito de manter o rigor metodológico e evitar conclusões errôneas ${ }^{10}$.

Inicialmente foi realizada uma pesquisa bibliográfica eletrônica exploratória para desenvolver e otimizar uma estratégia de busca e estimar a extensão e a qualidade dos estudos disponíveis de forma que abrangesse toda a literatura.

Em seguida, foi realizada uma busca integrativa da literatura nas bases de dados MEDLINE (PubMed), PEDRo e Livivo. Os termos de pesquisa foram: "multiple sclerosis", "specificity and sensitivity", "weights and measures", "scale", "score", "multiple sclerosis/complications" e "multiple sclerosis/physiopathology".

Após identificados os artigos, resumos foram lidos e em seguida o artigo foi lido na íntegra e selecionados de acordo com os critérios psicométricos (validade, reprodutibilidade, confiabilidade) e comparação com escalas ambulatoriais, 
quando o resumo ou artigo completo não atendeu ao tema de pesquisa, foi excluído.

\section{RESULTADOS}

No total, 373 resultados foram identificados ( 2 artigos foram excluídos por estarem duplicados); os resumos foram revisados e estruturados de acordo com os critérios psicométricos e em comparação com escalas ambulatoriais.

Quando o tema não atendeu à questão de pesquisa, o resumo foi excluído. Um total de 24 publicações foram classificadas como relevantes e foram lidas de forma integral, sendo excluídos artigos que não apresentavam no desfecho primário a validade das escalas ou não comparava a escala PDDS com graus de incapacidade. Finalmente, 7 artigos foram identificados como publicações metodológicas relevantes. A Figura 1 apresenta uma visão geral do processo de seleção.

Foram identificadas 7 publicações com conteúdo metodológico para PDDS. A maioria dos estudos foram publicados entre os anos de 1995 e 2019.

As características clínicas de cada estudo podem ser observadas no Quadro 1.

Para classificar os indivíduos em categorias de incapacidade leve, moderada e grave, a concordância entre EDSS e PDDS em alguns estudos teve forte correlação $\left(\rho=0,783^{11} ; \rho=0,711^{12} ; \rho=0,88^{13}\right.$ e $\left.\rho=0,723^{14}\right)$. 
Ao analisar a reprodutibilidade da escala PDDS, verificou-se que esta possui uma excelente reprodutibilidade teste-reteste $\quad(I C C=0,97 ; \quad I C 95 \% \quad 0,94-0,99)^{13} \quad$ e $(\mathrm{ICC}=0,911 \text {; IC95\% 0,685-0,918) })^{14}$ E uma boa confiabilidade entre a PDDS e a EDSS $(k=0,80)^{6},(k=0,93)^{13}$, $(k=0,58 ; p<0,001)^{14}$. Isto sugere que, quando usado em duas ocasiões diferentes separadas por um curto período de tempo, o autorrelato do nível de incapacidade no PDDS por pessoas com EM provavelmente será o mesmo.

Figura 1. Fluxograma do processo de seleção de publicações metodológicas.

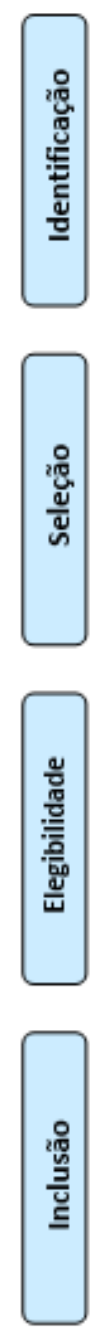

Registros identificados por meio de pesquisa no banco de dados ( $n=373$ )
Registros adicionais identificados por outras fontes $(n=2)$
Artigos de texto completo excluídos:

Não apresentar no desfecho as propriedades psicométricas $(n=8)$

Não apresentar comparação da escala PDDS com avaliações clínicas ambulatoriais $(n=2)$
Estudos incluídos no estudo

$(n=7)$

Artigos excluídos ( $n=349$ )

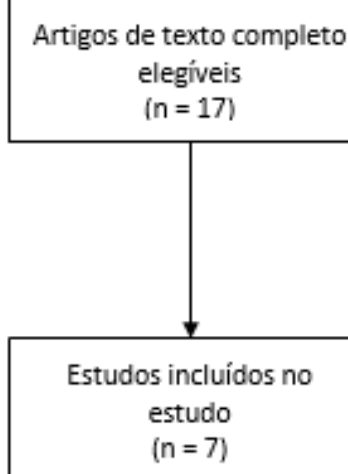
e 
Quadro 1. Características clinico-demográficas dos estudos selecionados.

\begin{tabular}{|c|c|c|c|c|c|c|c|c|c|c|c|}
\hline $\begin{array}{l}\text { Autor e } \\
\text { ano }\end{array}$ & $\begin{array}{l}\text { Tipo de } \\
\text { estudo }\end{array}$ & Local & Escala & $\begin{array}{l}\text { Tamanho } \\
\text { da } \\
\text { amostra }\end{array}$ & $\begin{array}{l}\text { Graus de } \\
\text { incapacidade }\end{array}$ & $\begin{array}{l}\text { Idade } \\
\text { (ano) }\end{array}$ & $\begin{array}{l}\text { Sexo } \\
\text { Feminino } \\
(\%)\end{array}$ & $\begin{array}{l}\text { Tempo de } \\
\text { diagnóstico } \\
\text { (ano) }\end{array}$ & EDSS & PDDS & NE \\
\hline $\begin{array}{l}\text { Hohol et } \\
a l, 1995^{6}\end{array}$ & Long & $\begin{array}{l}\text { Estados } \\
\text { Unidos }\end{array}$ & $\begin{array}{l}\text { EDSS } \\
\text { versus } \\
\text { PDDS }\end{array}$ & 1323 & $\begin{array}{l}\text { EMRR } \\
\text { EMPP } \\
\text { EMPS }\end{array}$ & 41,2 & 72,1 & - & 6 & 1 & II \\
\hline $\begin{array}{l}\text { Learmonth } \\
\text { et al, } \\
2013^{11}\end{array}$ & Transv & $\begin{array}{l}\text { Estados } \\
\text { Unidos }\end{array}$ & $\begin{array}{l}\text { EDSS } \\
\text { versus } \\
\text { PDDS }\end{array}$ & 96 & EMRR & 53,5 & 80 & 9 & 4,5 & 3,0 & II \\
\hline $\begin{array}{l}\text { Motl et al, } \\
2015^{15}\end{array}$ & Long & $\begin{array}{l}\text { Estados } \\
\text { Unidos }\end{array}$ & $\begin{array}{l}\text { PDDS } \\
\text { versus } \\
\text { MSWS- } \\
12\end{array}$ & 108 & EMRR & 47,1 & 88 & 10 & - & 3,7 & II \\
\hline $\begin{array}{l}\text { Lavorgna } \\
\text { et al, } \\
2017^{12}\end{array}$ & Transv & Itália & $\begin{array}{l}\text { PDDS } \\
\text { versus } \\
\text { EDSS }\end{array}$ & 96 & EMRR & 40 & 69,8 & 6 & 2 & 2 & II \\
\hline $\begin{array}{l}\text { Kister et } \\
a l, 2018^{16}\end{array}$ & Long & $\begin{array}{l}\text { Estados } \\
\text { Unidos }\end{array}$ & $\begin{array}{l}\text { PDDS } \\
\text { versus } \\
\text { PMSSS }\end{array}$ & 1740 & - & 44,5 & 73,7 & 11,2 & - & 1,9 & II \\
\hline $\begin{array}{l}\text { David et } \\
a l, 2019^{14}\end{array}$ & Transv & Brasil & $\begin{array}{l}\text { EDSS } \\
\text { versus } \\
\text { PDDS }\end{array}$ & 58 & $\begin{array}{l}\text { EMRR } \\
\text { EMPP }\end{array}$ & 37 & 76,19 & 7 & 2,5 & 1 & II \\
\hline $\begin{array}{l}\text { Solá-Valls } \\
\text { et al, } \\
2019^{13}\end{array}$ & Transv & Espanha & $\begin{array}{l}\text { EDSS } \\
\text { versus } \\
\text { PDDS }\end{array}$ & 103 & $\begin{array}{l}\text { EMRR } \\
\text { EMPS } \\
\text { EMPP }\end{array}$ & 44,3 & 67 & 12,8 & 2,0 & 0,5 & II \\
\hline
\end{tabular}

Alguns estudos compararam a escala PDDS com algumas escalas ambulatoriais. O estudo de Lavorgna et a/ ${ }^{12}$ observou forte correlação entre a PDDS com os testes de caminhada de 6 Minutos $(6 \mathrm{MW})(\rho=-0,633)$, escala de caminhada de esclerose múltipla com 12 Itens (MSWS-12) $(\rho=0,853)$ e caminhada de 25 pés por hora (T25FW) $(\rho=0,556)$, correlação moderada entre a PDDS e as escalas Timed Up and Go test (TUG) $(\rho=0,479)$ e Teste de Modalidades de Dígitos de Símbolos (SDMT) $(\rho=-0,402)$, mas correlação pequena entre a PDDS com o Teste de adição 
de série auditiva estimulada (PASAT) $(\rho=-0,24)$. No estudo de Learmonth et $a{ }^{11}$ os escores da PDDS também foram fortemente correlacionados com avaliações ambulatórias ( 6 MW $(\rho=-704)$, T25FW $(\rho=0,627)$, TUG $(\rho=0,717)$ e MSWS$12(\rho=0,801)$, correlação moderada com o desempenho cognitivo no SDMT $(\rho=-0,409)$ e tiveram pequenas correlações com o desempenho cognitivo no PASAT ( $\rho=-$ $0,244)$. No estudo de David et al ${ }^{14}$, também se observou uma forte correlação da escala PDDS com os instrumentos ambulatoriais (TUG $(\rho=0,76)$, T25FW $(\rho=0,72)$, 6MWT $(\rho=-$ $0,74)$ e SDMT $(\rho=-0,114)$.

Ao analisar as mudanças longitudinais na capacidade de andar usando o MSWS-12 em uma coorte, verificou-se que não houve correlação entre os escores de mudança para o MSWS-12 e o PDDS nos primeiros 6 meses. No entanto, em 24 meses, mostrou-se que houve uma correlação pequena entre as escalas $(\rho=0,35)$, demonstrando que as mudanças no MSWS-12 não se correlacionam com mudanças nos escores da PDSS, sugerindo que a PDDS é insensível às mudanças quando relacionada à deambulação dos indivíduos com EM ${ }^{15}$. Em contrapartida, quando é realizada a análise da progressão da doença a curto prazo comparando a PDDS e a escala Patient-derived Multiple Sclerosis Severity Score (PMSSS) identificou-se que 0 escore médio do PDDS aumentou de $1,9 \pm 2,2$ para 2,3 2,2 ( $p<0,0001)$, enquanto - PMSSS permaneceu praticamente inalterado (PMSSS inicial $=3,71 \pm 2,73$; final $=3,81 \pm 2,76$; teste $t$ pareado $p=0,28)^{16}$. 


\section{DISCUSSÃO}

O presente estudo revisou as propriedades psicométricas da escala PDDS para avaliar a progressão da doença em EM e verificar se apresenta propriedades psicométricas adequadas para ser usada em pesquisas e na prática clínica para a população estudada.

A validade da escala PDDS está sendo estabelecida por diversos estudos ${ }^{6,11-16}$ com o objetivo de utilizar uma escala mais confiável a fim de quantificar melhor a incapacidade dos indivíduos e assim avaliar melhor a progressão da doença quando a escala EDSS não puder ser aplicada, como, por exemplo, quando não houver um profissional experiente disponível ou quando houver dificuldades na pesquisa de campo. Dessa forma a PDDS auxiliará no processo de plano terapêutico na prática clínica, uma vez que esta é altamente dependente do comprometimento da marcha, que é um dos componentes principais e mais frequentemente utilizado.

Com base na revisão integrativa da literatura, podemos perceber que a PDDS se correlaciona à EDSS. Isso significa que uma escala que é definida pelo médico (EDSS) concorda com a autoavaliação feita pelo paciente (PDDS) em relação aos graus de incapacidade na EM.

Além disso, a escala PDDS possui boa confiabilidade e validade, apresentando traduções e adaptações transculturais para diferentes idiomas incluindo o inglês ${ }^{6,11}$, italiano $^{12}$, espanhol ${ }^{13}$ e português ${ }^{14}$. E possui uma excelente consistência interna para quando um paciente relatar 0 resultado do impacto da EM na marcha e apresenta pequena 
correlação com a duração da doença. Assim como também possui uma aceitabilidade para questionários telefônicos ${ }^{13}$ similar às realizadas de forma convencional.

Contudo, a PDDS, assim como EDSS, é uma escala que tem como foco a avaliação do comprometimento da marcha como podemos observar a partir da sua correlação com instrumentos ambulatoriais ${ }^{11-14}$, sendo, portanto, necessária a utilização de outros instrumentos de avaliação para avaliar sintomas comumente relatados por pacientes com EM, incluindo a fadiga, depressão, ansiedade e comprometimento cognitivo.

A limitação do estudo foi não conseguir avaliar a sensibilidade à mudança da escala PDDS em intervenções terapêuticas para EM pois, até o momento, não foram encontrados ensaios clínicos que utilizaram tal escala como variável de desfecho.

\section{CONCLUSÃO}

Disponível em diversos idiomas, a PDDS é uma escala validada, reprodutível e com consistência interna para avaliar a deambulação e progressão da doença do paciente. Além de possuir boa correlação com os graus de incapacidade quando o paciente é avaliado pela escala EDSS sendo, portanto, uma alternativa viável para a complementação da EDSS quando esta não puder ser praticável, como nas seguintes situações: quando não for possível realizar uma avaliação face a face, quando não 
houver profissional experiente disponível para administração, por limitações de tempo na coleta de dados ou por dificuldade na pesquisa na comunidade. Sendo assim, esses resultados podem ser de extrema importância na prática clínica.

\section{AGRADECIMENTOS}

Os autores agradecem à Universidade de Brasília pelo apoio institucional.

\section{REFERÊNCIAS}

1. Heldner MR, Vanbellingen T, Bohlhalter S, Mattle HP, Muri RM, Kamm $\mathrm{CP}$. Coin rotation task: a valid test for manual dexterity in multiple sclerosis. Phys Ther 2014;94:1644-51.

https://doi.org/10.2522/ptj.20130252

2. Neves MAO, Mello MP, Dumard $\mathrm{CH}$, Antonioli RS, Botelho JP, Nascimento OJM, et al. Abordagem fisioterapêutica na minimização dos efeitos da ataxia em indivíduos com esclerose múltipla. Rev Neurocienc 2007; 15:160-5.

http://www.revistaneurociencias.com.br/edicoes/2007/RN\%2015\%20 02/Pages\%20from\%20RN\%2015\%2002-12.pdf

3. Miller DH, Leary SM. Primary-progressive multiple sclerosis. Lancet Neurol 2007;6:903-12. https://doi.org/10.1016/S14744422(07)70243-0

4.Meyer-Moock S, Feng Y-S, Maeurer M, Dippel F-W, KohImann T. Systematic literature review and validity evaluation of the Expanded Disability Status Scale (EDSS) and the Multiple Sclerosis Functional Composite (MSFC) in patients with multiple sclerosis. BMC Neurol 2014;14:58. https://doi.org/10.1186/1471-2377-14-58

5.Kurtzke JF. Rating neurologic impairment in multiple sclerosis: an expanded disability status scale (EDSS). Neurology 1983;33:1444-52. https://doi.org/10.1212/wnl.33.11.1444

6. Hohol MJ, Orav EJ, Weiner HL. Disease steps in multiple sclerosis: A longitudinal study comparing Disease Steps and EDSS to evaluate disease progression. Mult Scler 1999;5:349-54. https://doi.org/10.1177/135245859900500508

7.Lo AC, Hadjimichael O, Vollmer TL. Treatment patterns of multiple sclerosis patients: A comparison of veterans and non-veterans using 
the NARCOMS registry. Mult Scler 2005;11:33-40. https://doi.org/10.1191/1352458505ms1136oa

8. Kobelt G, Berg J, Atherly D, Hadjimichael O. Costs and quality of life in multiple sclerosis: A cross-sectional study in the United States. Neurology 2006;66:1696-702.

https://doi.org/10.1212/01.wnl.0000218309.01322.5c

9. Marrie RA, Cutter G, Tyry T, Vollmer T, Campagnolo D. Does multiple sclerosis-associated disability differ between races? Neurology 2006;66:1235-40.

https://doi.org/10.1212/01.wnl.0000208505.81912.82

10. Whittemore $\mathrm{R}$, Knafl $\mathrm{K}$. The integrative review: updated methodology. J Adv Nurs 2005;52:546-53.

https://doi.org/10.1111/j.1365-2648.2005.03621.x

11.Learmonth YC, Motl RW, Sandroff BM, Pula JH, Cadavid D. Validation of patient determined disease steps (PDDS) scale scores in persons with multiple sclerosis. BMC Neurol 2013;13:37. https://doi.org/10.1186/1471-2377-13-37

12. Lavorgna L, Sparaco M, Esposito S, MotL RW, Gallo A, Bisecco A, et al. Validity and reproducibility of the Italian version of the patient determined disease steps scale in people with multiple sclerosis. Mult Scler Relat Disord 2017;18:173-6.

https://doi.org/10.1016/j.msard.2017.09.027

13.Solà-Valls N, Vicente-Pascual M, Blanco Y, Solana E, Llufriu S, Martínez-Heras $\mathrm{E}$, et al. Spanish validation of the telephone assessed Expanded Disability Status Scale and Patient Determined Disease Steps in people with multiple sclerosis. Mult Scler Relat Disord 2019;27:333-9. https://doi.org/10.1016/j.msard.2018.11.018

14.David AC, Sasaki JE, Ramari C, Tauil CB, Moraes AG, Martins F, et al. Validation of the Brazilian version of the patient-determined disease steps scale in persons with multiple sclerosis. Mult Scler Relat Disord 2019;30:208-14. https://doi.org/10.1016/j.msard.2019.02.022

15. Motl RW, Putzki N, Pilutti LA, Cadavid D. Longitudinal changes in self-reported walking ability in multiple sclerosis. PLoS One 2015;10:e0125002. https://doi.org/10.1371/journal.pone.0125002 16. Kister I, Bacon TE, Cutter GR. Short-term disability progression in two multiethnic multiple sclerosis centers in the treatment era. Ther Adv Neurol Disord 2018;11:1756286418793613. https://doi.org/10.1177/1756286418793613 